\title{
La nuova legge lombarda sul turismo
}

\author{
The new Lombardy law on tourism
}

Vincenzo Franceschelli e Sara D'Urso*

L'articolo traccia una panoramica articolata dei contenuti della recente legge regionale lombarda sul turismo, analizzando ed approfondendo gli aspetti maggiormente rilevanti della nuova normativa e mettendone in luce le potenzialità per lo sviluppo del turismo in Lombardia.

The article outlines a structured overview of the contents of the recent Lombardy law on tourism, analyzing and deepening the key aspects of the new legislation and highlighting the potential for the development of tourism in Lombardy.

\section{La nuova legge lombarda sul turismo}

In sintesi, si poterebbe dire che la nuova legge regionale 1 ottobre 2015 n. 27, denominata "Politiche regionali in materia di turismo e attrattività del territorio lombardo", vuole regolare un turismo accogliente, integrato, trasparente e tecnologico.

Accogliente, perché è diretta all'accoglienza, e alla fruizione dei servizi turistici.

Integrato, perché vuole favorire l'integrazione tra imprese - reti, consorzi, cooperazione - semplificando modalità e procedure, e offre al turista servizi integrati con i Trasporti e la Sanità lombardi.

Trasparente, perché richiede agli Enti e alle Imprese completezza di dati e informazioni al fine di avere un turista informato.

Tecnologico, perché valorizza ed utilizza le nuove tecnologie di informazione e trasmissione dati, in sintonia con l'Agenda digitale lombarda; e perché ricorda che la Lombardia è una regione avanzata ad alta tecnologia. Le novità sono, rispetto alla legge precedente, le seguenti:

a) un ribaltamento di prospettiva: della disciplina dei singoli istituti a connotazione turistica al tratto unitario della "accoglienza". Il diritto del turismo è visto come un insieme di norme dirette a migliorare l'accoglienza nella regione, favorendo la fruizione dei servizi turistici. b) Trasparenza e informazione. Per migliorare l'accoglienza e favorire la fruizione, il turismo, inteso come sistema, deve essere trasparente così da fornire al turista informazioni chiare e non decettive.

c) Ricorso alle nuove tecnologie. Il turista si affida sempre di più alle nuove tecnologie per scegliere $\mathrm{i}$ servizi turistici. La nuova legge ne tiene conto, operando scelte e regole compatibili con la piattaforma digitale.

d) Integrazione. La nuova legge si basa sul principio dell'integrazione. Integrazione nell'accoglienza; integrazione nei servizi; integrazione con l'agenda digitale.

1. Accoglienza integrata: la nuova legge favorisce la costituzione di sinergie di rete per la creazione e la gestione di servizi turistici integrati (partenariato, distretti).

2. Integrazione con i servizi regionali di eccellenza: si cerca, per la prima volta, di integrare i servizi turistici con il sistema regionale dei trasporti e della sanità.

3. Integrazione con il "turismo digitale": la nuova legge si coordina con il programma dell'agenda digitale lombarda, favorendo l'utilizzo della piattaforma digitale e dei servizi digitali.

e) Semplificazione e partecipazione. Si favorisce forse per la prima volta in un contesto turistico - il ricorso a strumenti di autodisciplina. 


\section{L'iter}

Con l'obiettivo di rivedere in toto il precedente Testo Unico in materia di turismo (legge regionale 16 luglio 2007, n. 15, Testo Unico delle leggi regionali in materia di turismo), la Regione Lombardia ha promulgato la nuova legge regionale che tiene conto del mutato contesto legislativo nazionale, comunitario e internazionale in materia turistica.

Come è noto, negli otto anni trascorsi tra l'entrata in vigore del precedente TU e la nuova legge diversi provvedimenti normativi sono stati emanati che hanno modificato e influenzato l'attuale settore. In un contesto di generale cambiamento la Regione Lombardia si è trovata di fronte ad un momento di grande attenzione nei confronti del turismo, anche grazie ad avere ospitato proprio nel proprio capoluogo di provincia l'Esposizione Universale 2015.

L'entrata in vigore del Codice del Turismo (d.lgs. 79/2011) ha avuto non poca rilevanza, sebbene sia stato dichiarato incostituzionale nella quasi totalità del contenuto di natura amministrativa dalla sentenza 5 aprile 2012 n. 801. Esso ha comunque rappresentato un tentativo di superamento di alcuni problemi di disomogeneità della disciplina di taluni istituti, e al contempo ha avviato il processo di abrogazione di alcune norme in materia turistica ${ }^{2}$. Altrettanto rilevante è la Legge 7 aprile 2014 n. 56 «Disposizioni sulle città metropolitane, sulle province, sulle unioni e fusioni di comuni», sul ruolo e le competenze degli enti territoriali, dalla quale il legislatore lombardo non ha potuto prescindere nel ridisegno delle competenze degli enti.

La Regione non ha perso l'occasione per rinnovare la disciplina del settore ponendosi come obiettivo principale, quale strumento per il raggiungimento di tutti gli altri, la semplificazione.

Il tema della semplificazione compare all'art. 2 lettera g) della legge 27 nel quale si statuisce che la Regione attua (non favorisce o attiva) politiche di semplificazione amministrativa per le imprese del turismo e, più in generale, della catena dell'attrattività, quindi di qualunque ente che entri a fare parte della filiera in grado di generare attrattività turistica ${ }^{3}$.

Nell'articolo rilevano due aspetti significativi il primo attiene alla definizione di impresa turistica e di filiera e il secondo alla semplificazione. Come è noto la definizione di impresa in senso turistico, dopo che per anni è stata identificata e circoscritta alle sole attività che erano spiccatamente turistiche, tanto da essere iscritte in una sezione separata del registro delle imprese, quali le strutture ricettive e le agenzie di viaggio e turismo ${ }^{4}$, grazie alla legge 135 del 2001, che avrebbe dovuto riformare la precedente legge quadro (217/83), è stata riscritta riproponendo sostanzialmente quanto disposto dell'art. 2082 del codice civile, a patto che l'attività di impresa, qualunque essa fosse, contribuisse all'offerta turistica dei sistemi locali, dalla stessa legge 135/01 introdotti. Quale fosse la ratio normativa non è difficile da interpretare: di fronte ad un ampliamento continuo dell'offerta turistica e degli operatori del settore, ma soprattutto di fronte alla difficoltà, anche a fini economici, di qualificare un'impresa come esclusivamente turistica o non turistica, il legislatore ha fatto una scelta più efficace incamerando all'interno delle imprese turistiche, tutte quelle che contribuissero in qualche modo alla formazione dell'offerta di servizi o prodotti per il viaggiatore.

La definizione di impresa dell'art. 7 della legge 135 del 2001 è stata così trasfusa nel codice del turismo all'art. 4 che, definendo un istituto privatistico, è sopravvissuto all'abrogazione da parte della Corte Costituzionale (sent. n. 80 del 2012) ${ }^{5}$. La scelta del legislatore lombardo entra in questo solco e all'art. 27 fa implicitamente proprio il concetto di impresa del turismo quale qualunque impresa che concorra a qualunque livello alla formazione della filiera turistica, per poi esplicitare la defi-
1. Corte. Cost., 5 aprile 2012, n.80, in Giust. civ., 2012, I, 1388, anche in Riv. it. dir. tur., 6/2012, p. 29 e ss., con nota di L. Righi. In seguito alla riforma del titolo $\checkmark$ della costituzione (legge di revisione costituzionale 18 ottobre 2011, n. 3) e la conseguente attribuzione della competenza legislativa esclusiva alle regioni in materia turistica, pochi sono gli ambiti residui allo Stato, principalmente convergono nell'ambito del Diritto privato. II codice del turismo, a parere della Corte, è risultato incostituzionale, non solo per la materia trattata, ma anche per il metodo: una delega al Governo in tema di semplificazione normativa. Per un'analisi più approfondita in materia di competenze in materia turistica si veda: M. Malo - C Colaluca, Competenze normative e fonti, in (a cura di V. Franceschelli - F. Morandi) Diritto del turismo, Torino, 2013, p. 21 e ss.

2. Per quanto riguarda l'intervento statale in materia turistica la Consulta ha sì cassato buona parte del Codice del turismo, ma d'altra parte ha stabilito in diverse pronunce che questo è ammissibile quando sia motivato, proporzionato, ma soprattutto si fatto d'intesa con le Regioni, proprio questo, infatti, è puntun dolens che viene contestato al Codice. Sempre sotto il profilo delle competenze si veda Corte Cost., 24 giu- gno 2005, n. 242, in Giur. it., 2006, p. 682; Corte Cost. 5 marzo 2007, n. 88, in Dir. tur., 2007, p. 274 e ss., con nota di S. Piazza; Corte Cost., 12 ottobre 2007, n. 339, in Foro it., 2008, I, p. 396; Corte Cost., 20 marzo 2009, n. 76. Tra le norme abrogate dal codice del turismo: legge 135 del 2001, art. 4 d.I. n. 7 del 2007 per l'adeguamento della disciplina delle guide turistiche degli accompagnatori ai principi comunitari: la legge 27 dicembre 1977, n. 1084, che ha reso esecutiva la Convenzione internazionale sul contratto di viaggio (CCV) del 23 aprile 1970, abrogazione sottoposta alla denuncia dello Stato italiano, non ancora pervenuta al momento in cui si scrive.

3. II processo di semplificazione degli adempimenti amministrativi delle strutture turistico-ricettive già nella precedente legge era conforme a quanto stabilito dalI'articolo 19 della legge 7 agosto, n. 241 per quanto attiene alla segnalazione certificata di inizio attività.

4. Come indicato alla Legge quadro $217 / 83$.

5. Per un'analisi dell'impresa turistica nella 135 del 2001 e nel codice del turismo si veda: L. Righi, Le strutture ricettive, in (a cura di V. Franceschelli - F. Morandi) Diritto del turismo, Torino, 2013 p. 132. 
nizione nel successivo articolo 70. Qui ricalca la dizione codicistica per la prima parte della definizione, ma omette l'ultimo frammento del primo comma dell'art.4 del codice. Sparisce così l'appartenenza all'"offerta turistica" a favore della "filiera turistica", quale marcatore per identificare un'impresa come tale viene al contempo incrementato, al comma 2, l'elenco delle possibili attività facenti parte dell'“attrattività territoriale": somministrazione di alimenti e bevande, del tempo libero, dello sport e del benessere, culturali e ricreative, delle tecnologie digitali, di valorizzazione della produzione agroalimentare e artigianale regionali, del commercio al dettaglio con particolare riferimento a quelle storiche e di qualità.

Postulando questo, la semplificazione attuata dalla norma si estende a qualunque impresa che nel territorio lombardo, ma non solo, decida di intraprendere o continuare un'attività di natura turistica, in qualunque comparto del settore stesso. All'articolo 27, infatti, dopo avere dedicato la lettera $\mathrm{p}$ ) al turismo delle famiglie in generale, la lettera q) al turismo religioso, alla lettera $u$ ) stabilisce l'obiettivo della valorizzazione del turismo d'affari, congressuale, fieristico, sanitario, industriale, culturale, sportivo ed enogastronomico, esplicitando già in questa sede, quindi, tutti i comparti possibili nei quali si articola il turismo lombardo, coerentemente con la realtà del tessuto socio-economico. A corollario di questa concezione costitutiva dell'offerta e della filiera turistica, in cui ogni elemento che ne faccia parte è attratto dalla caratteristica della "turisticità", viene introdotto all'articolo 48 l'impianto turistico, quale nuovo elemento della filiera. Dal punto di vista della qualificazione giuridica non viene data una definizione dello stesso, molto probabilmente a volere permettere la massima flessibilità all'operatore del settore (impresa, associazione...) ciò che qui interessa è l'attribuzione della qualifica turistica a quelle strutture materiali (quali stadi, teatri, auditorium) che siano funzionali all'attrattività del territorio e permettano lo svolgersi di attività esperienziali che, per dimensione e caratteristiche siano "attrattori di flussi".

La semplificazione che attua la nuova legge non si limita ad un processo rivolto alle imprese, per le quali sono

6. Sul tema della semplificazione normativa si veda: A. Natalini, /l tempo delle riforme amministrative, Bologna, 2006, pag. 179: "All'inizio degli anni Novanta I'ordinamento giuridico italiano era afflitto da un'inflazione normativa che influiva negativamente sul funzionamento delle pubbliche amministrazioni e sulle attività dei privati».

7. Cfr. S. Cassese, Istituzioni di diritto amministrativo, Milano, 2004, p. 209 «Naturale derivazione dei principi di buon andamento, economicità ed efficacia è quello di semplicità. Esso ha varie esplicazioni, relative all'organizzazione amministrativa, alla gestione delle risorse umane e finanziarie e allo svolgimento delle appunto previste facilitazioni burocratiche, ma si manifesta anche nello stesso testo normativo dove viene operata una riorganizzazione dell'articolato in un'ottica di semplificazione e chiarezza ai fini del fruitore ${ }^{6}$.

Dell'importanza della semplificazione quale mezzo per una crescita del settore ci si era già resi conto sia a livello regionale sia a livello statale ${ }^{7}$, tanto che l'articolo 16 del codice del turismo, articolo ora abrogato, titolava proprio Semplificazione degli adempimenti amministrativi delle strutture turistico-ricettive.

La questione della semplificazione si inserisce in una politica in tal senso, che fino al 2001 con la riforma del titolo V della Costituzione, si è manifestata principalmente sul piano nazionale, a partire dal 2001 ha spostato il suo baricentro dal piano nazionale a quello regiona$\mathrm{le}^{8}$, conservando al contempo un interesse generale, anche per il forte impulso di derivazione comunitaria. L'Unione europea, infatti, con la direttiva 2006/123/CE, attuata in Italia con il d.lgs. 26 marzo 2010, n. 59 ha posto l'obiettivo di eliminazione di regimi autorizzatori, formalità burocratiche che siano da ostacolo, perché eccessivi, all'intraprendere nuove attività e ha fissato al contempo il principio dell'unico interlocutore con il quale svolgere tutti gli adempimenti necessari, utilizzando prevalentemente mezzi telematici.

Liter semplificativo può essere così riassunto: un primo provvedimento fu la legge 241 del 1990, Nuove norme in materia di procedimento amministrativo $e$ di diritto di accesso ai documenti amministrativi, oggetto di recente revisione, un secondo l'art. 2 della legge n. 537 del 1993, che introduceva nuovi criteri di semplificazione procedimentale. Merito di questa legge è non solo di avere attuato un'ampia delegificazione dell'azione amministrativa, ma soprattutto di avere introdotto i criteri di razionalizzazione e uniformazione di procedure anche di competenza di amministrazioni diverse e di avere liberalizzato alcune attività private, nonché esteso i casi di silenzio assenso ${ }^{9}$. Successiva al provvedimento del 1993 è la c.d. "legge Bassanini", legge n. 59 del 1997, che operava una modifica integrativa dell'art. 17 della legge n. 400 del 1988 e che disciplinava il potere regolamentare del Governo. È seguito poi un periodo teso al miglioramento della qualità delle

funzioni»; S. Amorosino, La semplificazione amministrativa e le recenti modifiche normative alla disciplina generale del procedimento, in Foro amministrativo-Tar 2005, VII-VIII, p. 2635: "La tematica delle semplificazioni amministrative va inquadrata nella più ampia problematica della modernizzazione amministrativa del nostro Paese, da cui - a sua volta - dipende in rilevante misura la competitività».

8. Nel solo settore del turismo si vedano: legge Reg. Veneto 14 giugno 2013, n.

11; legge Regione Umbria 12 luglio 2013, n.13; legge Regione Lazio 6 agosto 2007, n. 13.

9. Si veda V. Cerulli Irelli, Corso di diritto amministrativo, Torino, 1994, p. 462. 
norme, quindi della loro chiarezza e misura ${ }^{10}$. A seguire, tra gli altri, il provvedimento relativo allo sportello unico di cui all'articolo 38 del decreto-legge 25 giugno 2008, n. 112 Disposizioni urgenti per lo sviluppo economico, la semplificazione, la competitività, la stabilizzazione della finanza pubblica e la perequazione tributaria, convertito con modificazioni dalla legge 6 agosto 2008 n. 133 e del relativo regolamento attuativo, richiamato direttamente dalla legge lombarda, che alla semplificazione degli adempimenti a carico degli imprenditori per agevolarne l'inizio attività o variazioni in corso, ha affiancato la semplificazione dell'articolato stesso con l'obiettivo della sintesi e della chiarezza dei principi. E stato così ridotto il numero totale delle norme, grazie all'operazione di accorpamento di alcune di queste che dettavano principi comuni a più istituti. Ne è di esempio il titolo relativo alle strutture ricettive. Nel titolo III, infatti,viene mantenuta la bipartizione tra strutture ricettive alberghiere e non alberghiere (diversamente elencate), ma vengono accorpate nel capo IV dall'articolo 37 al 41 tutte le disposizioni comuni alle due categorie; vengono poi eliminati quasi completamente $\mathrm{i}$ rimandi diretti alle leggi vigenti (numero e anno) in favore di richiami "alla disciplina attualmente in vigore”, al fine di evitare provvedimenti di aggiornamento della norma.

\section{Sintesi dei punti qualificanti della legge}

Oltre alla semplificazione, che rappresenta una delle principali finalità della norma, all'articolo 2 viene indicato l'insieme degli obiettivi, che trovano riscontro diretto nell'articolato:

- Valorizzazione e sviluppo della filiera dell'attrattività intesa come sistema integrato, per cui si prevede la stesura triennale di un piano (art. 15) che, sulla base dell'analisi del contesto internazionale, nazionale dei trend del settore, dello stato di fatto delle politiche attivate dalla Regione negli anni precedenti, individua gli obiettivi e le linee principali di intervento.

- Forte integrazione tra soggetti pubblici e privati allo scopo di valorizzare la filiera attraverso la costruzione di servizi turistici innovativi e di qualità e nuovi investimenti. Sono infatti previsti, tra gli altri, all'art. 72 interventi per l'ammodernamento, il potenziamento e la qualificazione delle strutture e infrastrut- ture e all'art. 82 forme di contribuzione ed agevolazione per programmi, progetti e iniziative di investimento per la diversificazione e il potenziamento delle infrastrutture pubbliche, destinate ad un utilizzo anche ai fini turistici e dell'attrattività.

- Spinta alle aggregazioni di imprese sia attraverso reti, sia attraverso i contratti di consorzio o cooperative, funzionali ad una crescita dell'attrattività.

- Valorizzazione del capitale umano: si riconosce la criticità di un alto livello di competenza e conoscenza, quale strumento per rendere più competitiva l'offerta di prodotti turistici; a tal fine si favorisce l'educazione e la formazione della forza lavoro. Finalità che viene declinata all'articolo 77 in cui si prevede che la Giunta, coerentemente con le azioni individuate nel piano per lo sviluppo del turismo e dell'attrattività (ex art. 15) e nel piano annuale ( $e x$ art. 16), promuove e sostiene la valorizzazione del capitale umano, la qualificazione delle risorse professionali, l'alta formazione e la formazione continua quali valori ed elementi strategici per la crescita e lo sviluppo del turismo e dell'attrattività del territorio.

- Valorizzazione del ruolo delle associazioni di categoria non solo appartenenti al settore del turismo, ma anche a settori direttamente collegati a questo (commercio, artigianato...) e attivazione di accordi di collaborazione interistituzionali con enti pubblici e privati.

- Valorizzazione turistica del territorio attraverso accordi anche interregionali, riprendendo un tema già presente nel precedente TU all'articolo 4, dei sistemi turistici.

- Incentivazione alla digitalizzazione integrata pubblico-privato volta anche alla commercializzazione dei servizi. Tradotta all'articolo 76 , dove sono previste forme di contribuzione e agevolazione a favore delle imprese turistiche (ex art. 70) al fine di sostenere la competitività del turismo e dell'attrattività territoriale, in coerenza con l'agenda digitale lombarda.

- Accessibilità del turismo.

- Sostenibilità ed ecosostenibilità del turismo. L'Organizzazione mondiale del turismo nel 1988 ha dato una definizione che cosa si intenda per turismo sostenibile: «le attività turistiche sono sostenibili quando si sviluppano in modo tale da mantenersi vitali in un'area turistica per un tempo illimitato, non alterano l'ambiente (naturale, sociale, artistico) e non ostacolano o inibiscono lo sviluppo di altre attività sociali ed economiche». La sostenibilità è un obiettivo non solo a 
livello regionale, ma soprattutto a livello nazionale e comunitario. La Regione Lombardia, da sempre attenta alla questione, prima della norma in commento aveva già emanato una provvedimento contenuto all'articolo 5 della precedente legge.

- Semplificazione della fruibilità del patrimonio turistico anche attraverso la Carta del turista che permetta la fruizione integrata dei servizi turistici, di trasporto e sanitari.

- Garanzia delle attività per la tutela dei diritti del turista a partire dalle modalità di conciliazione paritetica.

- Promozione dell'immagine unitaria della regione Lombardia all'estero mantenendo sempre l'indicazione del marchio "Italia".

\section{Integrazione tra soggetti pubblici e pri- vati}

Lobiettivo della integrazione tra soggetti pubblici e privati viene declinato all'interno della norma in diversi articoli. Al capo II «Strumenti e organismi di partecipazione», in particolare, gli artt. 8, 9, 10, trattano del partenariato quale mezzo per raggiungere le finalità indicate all'art. 2 e per conseguire la massima partecipazione dei soggetti pubblici e privati. Il testo al comma 3) dell'art. 8 stabilisce che queste libere aggregazioni di soggetti, per potere operare e beneficiare della promozione delle opportunità di investimento, dell'accesso al credito, della semplificazione e celerità nei rapporti con le pubbliche amministrazioni, dell'attribuzione dello svolgimento di attività che rivestono anche un interesse pubblico, è sufficiente che individuino tra loro un soggetto guida, identificato dalla Regione come capofila e vengano riconosciuti dalla Regione quali enti in grado di valorizzare destinazioni turistiche e aree esperienziali, quali ambiti dotati di risorse, infrastrutture e prodotti. Il legislatore regionale con l'espressione liberamente aggregati, ha volutamente lasciato autonomia ai soggetti di utilizzare la forma giuridica di aggregazione che meglio permetta loro il raggiungimento di obiettivi di efficacia ed efficienza. Al comma 2) dello stesso articolo, infatti, statuisce che possono essere stipulati accordi e convenzioni tra enti pubblici, reti di imprese ${ }^{11}$, consorzi turistici ${ }^{12}$, organizzazioni imprenditoriali, associazioni di categoria, associazioni dei consumatori iscritte nell'elenco regionale e altri soggetti della filiera dell'attrattività, quali i sistemi turistici.

Sempre nell'ambito della partecipazione all'articolo 10 vengono introdotti i distretti dell'attrattività del territorio e distretti del commercio, richiamando l'art. 5 delle legge regionale 2 febbraio 2010 n. 6 (testo unico delle leggi regionali in materia di commercio e fiere), che sono accordi della Regione con i comuni e con altri soggetti per la realizzazione di interventi e iniziative integrate per l'attrattività territoriale, turistica e commerciale. Nascono sulla falsariga dei già esistenti Distretti del commercio lombardo (declinati in DUC, distretto urbano del commercio, e DID, distretto diffuso di rilevanza intercomunale) e mirano a sfruttarne a livello turistico i benefici di integrazione, di coesione sociale e di valorizzazione del territorio. Il Distretto che, rispetto alle reti, non ha natura negoziale rappresenta un'ulteriore opportunità per il rilancio di aree turistiche fruendo delle forze di interlocutori di diversa natura, anche appartenenti a settori differenti che concorrano alla valorizzazione del territorio ${ }^{13}$. Al contempo scompare l'articolo sui sistemi che erano stati introdotti con la legge 135/2001, sostituiti dalle nuove forme di integrazione pubblico-privato.
11. La collaborazione tra imprese basata su rapporti contrattuali può manifestarsi attraverso: Associazione in partecipazione, patrimoni destinati a uno scopo, associazioni temporanee di imprese, consorzi, joint venture, reti di imprese, GEIE. Con il contratto di rete più imprenditori perseguono lo scopo di accrescere, individualmente e collettivamente, la propria capacità innovativa e la propria competitività sul mercato e a tal fine si obbligano, sulla base di un programma comune di rete a collaborare in forme e ambiti predeterminati attinenti all'esercizio delle proprie imprese ovvero a scambiarsi informazioni o prestazioni di natura industriale, commerciale, tecnica o tecnologica, ovvero ancora ad esercitare in comune una o più attività rientranti nell'oggetto della proprie impresa. Art. 42 , comma $2^{\circ}$ quater, legge 30 luglio 2010, n.122 prevede le agevolazioni fiscali per le reti di impresa. Art. 66, d.I. 22 giugno 2012, n. 83, convertito in legge 7 agosto 2012, n.134 prevede ulteriori incentivi. II contratto di rete rappresenta, indipendentemente dal turismo, una forma di coordinamento tra imprese agevolata dalla disciplina pubblicistica. Nel comparto turistico i programmi di rete incentivati devono risultare coerenti con progetti volti alla realizzazione di obiettivi specificamente individuat dal d.m. 8 gennaio 2013 in attuazione dell'art. 66 d.I. 83/2012, per consentire una rigorosa selezione delle iniziative finanziabili: realizzazione e perfezionamento di strumenti informativi di amministrazione, di gestione e di prenotazione de servizi turistici, creazione di piattaforme per l'acquisto collettivo di beni e/o servizi; diffusione della conoscenza del territorio a fini turistici, con particolare riferi- mento a sistemi di promozione e commercializzazione in via telematica; la predisposizione di pacchetti turistici innovativi; la promozione su mercati esteri mediante la partecipazione a fiere e la creazione di materiali promozionali comuni. Per un'analisi delle reti di impresa si veda D. Scarpa, La nuova impresa tra integrazione, patrimonialità e comunicazione, Napoli, 2011.

12. Per un'analisi generale del contratto di consorzio si veda: M. Sarale, Consorzi e società consortili, in G. Cottino - M. Sarale - R. Weigmann, Società di persone e consorzi, Milano, 2004, p. 407 e ss.; R. Franceschelli, Consorzi per il coordinamento della produzione e degli scambi art. c.c. 2602- 2620, in (a cura di Scialoja Branca) Commentario al codice civile, Bologna, 1992. La legge regionale veneto $11 / 2013$ all'articolo 18 istituisce i consorzi di imprese turistiche, quali associazioni, anche costituite nella forma di società consortile, formate da imprese turistiche e da eventuali altri soggetti privati.

13. I distretti del sono stati introdotti in Lombardia nel 2004. La legge 180 del 2011 ne ha dato una definizione a livello nazionale. L'art. 3 quarto comma d.I. 13 maggio 2011, n. 70, convertito in legge 12 luglio 2011, n. 106, ha abilitato le imprese turistiche operanti nei territori costieri a richiedere l'istituzione dei distretti turistici al fine di riqualificare e rilanciare l'offerta turistica a livello nazionale ed internazionale, di accrescere lo sviluppo delle aree e dei settori del Distretto, di migliorare l'efficienza nell'organizzazione nella produzione dei servizi e di assicurare sostegno alle imprese. 


\section{Valorizzazione del territorio lombardo e della sua attrattività}

L'articolo 3 disciplina la promozione e la valorizzazione dell'immagine del territorio lombardo. Coerentemente con il resto del testo normativo le modalità e gli strumenti che la Giunta dovrà individuare per promuovere la destinazione "Lombardia" non possono prescindere dalla collaborazione tra pubblico e privato, non solo per la promozione delle eccellenze, ma anche per la creazione di prodotti tematici e filiere di prodotto. La collaborazione e il mettere a fattor comune la risorse esistenti, indipendentemente dalla natura dei soggetti, corrisponde infatti allo strumento principe individuato per conseguire risultati di efficacia e di efficienza. Non è casuale, infatti, la previsione all'art. 16 del piano annuale della promozione turistica e dell'attrattività, con il chiaro intento, da parte della Regione di coordinamento e regia delle politiche promozionali allo scopo di garantirne unitariamente l'efficacia. Ulteriore interessante elemento di novità è indicato al quarto comma dello stesso articolo: la promozione della destinazione Lombardia e dei suoi prodotti nei mercati esteri deve sempre essere abbinata all'indicazione "Italia". La stessa scelta è stata fatta poco tempo prima dalla Regione Veneto, legge regionale 14 giugno 2013, n. 11 Sviluppo e sostenibilità del turismo veneto, che all'art. 5 comma 2, specifica che il marchio "Veneto" nei mercati esteri è sempre affiancato dall'indicazione Italia. L'aver reso obbligatoria l'indicazione "Italia”, ogniqualvolta la destinazione Lombardia venga promossa nei mercati esteri, garantisce la riconoscibilità del marchio lombardo, evita la dispersione di risorse tutte quelle volte in cui all'estero il marchio regionale non sia, purtroppo, ancora riconosciuto e soprattutto limita il verificarsi di fenomeni di confusione tra i potenziali destinatari del messaggio ${ }^{14}$. La possibilità per le Regioni di svolgere attività promozionale all'estero era prevista dall'art. 4 del d.lgs. n. 616 del 1977, modificato dall'articolo 8 della legge n. 59 del 1997 ed è stata confermata ed ampliata in seguito alla riforma costituzionale (art. 117 quarto comma), ciò comporta una necessaria maggiore attenzione da parte dei diversi legislatori regionali di fronte al rischio di azioni promozionali disomogenee e non coordinate tra regioni. Non va sottovalutato che in questo contesto normativo il ruolo dell'ENIT, quale prin- cipale veicolo del messaggio pubblicitario dall'Italia all'estero sta via via assottigliandosi.

\section{Protezione del turista viaggiatore e turi- smo accessibile}

Per quanto attiene alla accessibilità la disciplina è contenuta all'art. 4 che stabilisce che obiettivo della Regione è assicurare, quindi garantire, una fruizione completa ed autonoma che garantisca gli stessi livelli di qualità allo stesso prezzo, alle persone con disabilità motorie, sensoriali, e intellettive ${ }^{15}$. L'articolo è ripreso senza modifiche dal testo dell'articolo 3 del Codice del turismo, articolo abrogato in quanto, ha affermato la Corte Costituzionale, attiene con evidenza ai rapporti Stato Regioni, in materia di turismo e realizza un accentramento di funzioni che, sulla base della natura residuale delle competenze legislative regionali spettano in via ordinaria alle regioni salvo che lo Stato non operi l'avocazione delle stesse. La ripresa della norma nazionale pare coerente al contesto legislativo nazionale ed europeo in materia di disabilità dal quale, sebbene abbia la competenza assoluta, la Regione non può prescindere. Quindi interviene all'art. 74 prevedendo una serie di contribuzioni e agevolazioni (di cui all'articolo 71) per favorire il giusto abbattimento delle barriere architettoniche e accrescere la fruibilità turistica ai soggetti con disabilità motorie. Ne è un esempio il comma 10 dell'articolo 38 dove si prevede che la Giunta promuove e favorisce le strutture ricettive che applicano le norme vigenti in materia di accessibilità in base alla categoria urbanistica di appartenenza e che offrono servizi, strutture aggiuntive e standard oltre agli obblighi di legge, per incrementare l'accessibilità e migliorare l'accoglienza delle persone con disabilità motorie, sensoriali, intellettive.

\section{Organismi di informazione e coordina- mento}

Il capo III che va dall'art. 11 all'art. 14 tratta degli organismi di informazione e coordinamento. Il primo degli articoli si occupa delle Strutture di informazione e accoglienza turistica che furono istituite attraverso le APT con la prima legge quadro in materia turistica, la 217 del 1983 all'art. $4^{16}$. Nella nuova legge vengono in
14. Per un'analisi del tema della promozione turistica a livello regionale si veda $\mathrm{L}$. Degrassi (a cura di L. Degrassi - V. Franceschelli), Turismo diritto e diritti, Milano, 2009, p. 166 e ss.

15. Per uno studio sulla disabilità si veda S. Assennato - M. Quadrelli, Manuale della disabilità, Maggioli, 2012.
16. Per un quadro riepilogativo delle diverse esperienze regionali su APT e IAT, si veda M. Malo e A. Perini, Promozione, informazione, accoglienza turistica, in (a cura di V. Franceschelli - F. Morandi) Diritto del turismo, Torino, 2013, p. 74 e ss. 
primis individuati i criteri che devono essere rispettati da ciascun ufficio di informazione su tutto il territorio regionale, in particolare si tratta di imparzialità, omogeneità, trasparenza, qualità, professionalità e pari rappresentatività di tutto il territorio e della sua offerta. La Giunta stabilisce quindi i criteri per la istituzione delle strutture, nonché la loro localizzazione minima necessaria per garantire un'adeguata copertura territoriale. Alle sedi storiche, tra cui aeroporti e stazioni ferroviarie, si aggiunge la possibilità di creare IAT anche presso le principali strutture stradali ed autostradali. Una regia accentratrice degli uffici si è manifestata parimenti nella scelta della Regione Veneto dove è stato istituito il Piano d'azione per la realizzazione di una rete regionale di uffici di informazione che sia collegata pure a livello informatico anche con l'obiettivo di migliorare la circolazione delle informazioni per la soddisfazione del turista ${ }^{17}$.

Sotto il profilo delle novità introdotte sono state ampliate le attività che gli uffici sono autorizzati a svolgere, non solo informazione ed eventualmente prenotazione di servizi, ma vera e propria vendita di biglietti (per la fruizione dell'offerta e dei trasporti). Sotto la responsabilità degli IAT è stato introdotto anche un efficace strumento di misurazione diretta della soddisfazione del turista in relazione ai servizi fruiti, che è il "questionario di gradimento", predisposto su di un modello unico per tutta la regione.

Si richiede pertanto alle strutture già presenti nel mercato di provvedere ad un adeguamento entro 120 giorni dalla approvazione della deliberazione di Giunta.

Per quanto attiene alle associazioni pro-loco, che sono enti di natura privatistica, senza fini di lucro nati nel 1881, che svolgono la propria attività di valorizzazione delle realtà e potenzialità turistiche, naturalistiche, culturali, storiche, sociali, enogastronomiche de luoghi in cui operano, mantengono nella sostanza la natura e gli scopi già previsti nel precedente TU, anche se formalmente la disciplina è stata sintetizzata tutta in un unico articolo. $\mathrm{Si}$ iscrivono all'albo regionale istituito presso la Giunta.

\section{Nuovi strumenti per le politiche turisti- che e dell'attrattività: il tavolo regionale e l'Osservatorio}

All'articolo 13 è prevista l'istituzione di un tavolo regionale per le politiche turistiche, la funzione è assicurare il coordinamento tra i soggetti partecipanti senza gravare sul bilancio regionale. Altre regioni hanno previsto con finalità analoghe un tavolo di consultazione, la Regione Campania ha istituito all'art. 6 della Legge Regionale n. 18 dell'8 agosto 2014, un tavolo istituzionale per le politiche turistiche che svolge un'attività di natura consultiva ${ }^{18}$. Anche in questo contesto il legislatore lombardo ha preferito rimandare ad una delibera della Giunta la definizione le modalità di funzionamento, nonché i componenti del tavolo. La scelta si dimostra coerente con il progetto di semplificazione della norma, nonché di maggiore flessibilità della stessa.

All'articolo 14 viene istituito l'Osservatorio regionale del turismo, esperienza già presente in altre regioni ${ }^{19}$. L'osservatorio rappresenta il veicolo attraverso il quale poter conseguire diverse delle finalità indicate all'articolo 2. Attraverso l'analisi dei dati, raccolti prevalentemente per via telematica, la Regione non solo potrà verificare ed analizzare i flussi turistici interni confrontandoli con standard di mercato, ma avrà anche modo di monitorare e valutare l'impatto degli interventi realizzati a seguito delle politiche regionali sul territorio, quindi di verificare l'efficacia e i risultati delle azioni poste in essere. L'Osservatorio, rappresentando il centro di raccolta di dati di natura turistica provenienti da diverse fonti (comprese le SCIA che riceve dai comuni, nonché le rilevazioni attraverso i questionari di gradimento somministrati direttamente ai turisti) ne permette una contestuale analisi ed un utilizzo incrociato.
17. Giunta Regione Veneto deliberazione n. 2452 del 16 settembre 2008. La regione Veneto con la legge regionale n. 14 giugno del 2013 all'articolo 15 ha stabilito che la Giunta regionale dovrà indirizzare e coordinare le attività e definire gli standard minimi di informazione e accoglienza.

18. Le legge Regione Veneto 14 giugno 2013, n. 11 ha istituito all'articolo 10 Comitato regionale per le politiche turistiche. Anche la Legge Regione Umbria 12 luglio 2013, n. 13 all'articolo 12 ha previsto un comitato di coordinamento per la promozione turistica e integrata.

19. La Regione Lazio con la Legge regionale n. 13/2007 (Organizzazione de sistema turistico laziale), art. 20, ha istituito, presso l'Assessorato regionale competente in materia di turismo, l'Osservatorio regionale del Turismo, per monito- rare il settore turistico attraverso l'acquisizione, la gestione e la diffusione delle informazioni e dei dati statistici sui flussi tra domanda e offerta turistica regionale. Tra le altre regioni si vedano: Regione Marche legge 4 luglio 2006 n.9 prevede I'Osservatorio alla'art. 4; legge Regione Piemonte 11 febbraio 2002 n.1; legge Regione Umbria del 12 luglio 2013 n. 13 all'art. 11, legge Regione Veneto del 14 giugno 2013 n. 11 all'art. 14. La legge regionale n. 17/2011 (Bollettino Ufficiale della Regione Lazio n. 48 del 28/12/2011) ha modificato gli artt. 28 e 31 della legge Regionale n. 13/2007, prevedendo I'obbligatorietà, da parte degli operatori, della trasmissione telematica dei dati sui flussi turistici, in particolare su arrivi e presenze nelle strutture ricettive. Con delibera di Giunta Regionale $n$. 146 del 10/04/2015, sono stati individuati compiti e modalità di funzionamento dell'Osservatorio. 


\section{Ricettività turistica}

Il titolo III in materia di ricettività turistica presenta notevoli elementi di riforma sia nel contenuto delle norme, sia nell'organizzazione delle stesse. Ai fini della semplificazione, come accennato più sopra, tutti i principi comuni alle diverse tipologie di strutture ricettive sono stati accorpati in articoli di sintesi che ricomprendono qualunque struttura rientri nella disciplina. Novità sono state apportate anche alle categorie di strutture ricettive che vedremo nel dettaglio. La ratio sottostante la revisione e la sintesi delle tipologie ricettive risiede nell'eliminazione di tutti quei complessi che, al di là del contenuto definitorio, non presentano elementi di qualificazione giuridica per cui meritino una disciplina a sé stante. In questa prospettiva sono pertanto sparite dall'elenco delle strutture ricettive alberghiere le dimore storiche, le beauty farm. Per la ragione inversa trovano spazio nella norma i condhotel. In ottemperanza a quanto previsto dalla direttiva europea sui servizi si conferma l'utilizzo della SCIA (acronimo per Segnalazione Certificata di Inizio Attività) ${ }^{20}$, quale unico adempimento necessario per poter intraprendere o modificare un'attività ricettiva che va presentata esclusivamente allo Sportello Unico SUAP, esclusivo destinatario della documentazione richiesta in formato telematico ${ }^{21}$, come già previsto agli artt. 16 e 17 del codice del turismo ora abrogato.

Interessante e complessa novità è l'introduzione, all'art. 41 dell'obiettivo di integrazione e concentrazione dei controlli volti a verificare l'ottemperanza alle norme. L'enorme beneficio che si otterrebbe riuscendo a concentrare tutti i controlli in uno stesso momento, sarebbe sia a vantaggio dell'operatore che potrebbe ridurre le ore di lavoro perse, perché dedicate a fornire informazioni e documenti a chi svolge i controlli, ma anche per i controllori che potrebbero mettere a fattore comune, quindi beneficiare dell'utilizzo di informazioni già in possesso di altre amministrazioni (come previsto dalla legge regionale 19 febbraio 2014, n. 11). Tutte le strutture ricettive sono infatti tenute al rispetto delle normative vigenti in materia fiscale, e di sicurezza ${ }^{22}$, quindi passibili di controlli ed eventuali sanzioni (artt. 39, 40). La

20. Rimangono validi i limiti previsti all'art. 19 della legge 7 agosto 1990, n. 241 , tra cui immigrazione, sicurezza, difesa nazionale.

21. Art. 2 d.P.R. 160 del 2010.

22. Nel testo normativo sono spariti per quanto possibili i riferimenti diretti a testi di legge, rimandando invece a le "norme vigenti" si evita in un futuro di dovere procedere a continue revisioni ed aggiornamenti normativi.

23. Per un'analisi delle disciplina pubblicistica delle strutture ricettive: M. Gola - A

Zito - A. Cicchetti, Amministrazione pubblica e mercato del turismo, Milano,

2012; M. Gola - T. Groppi, Diritto pubblico del turismo, Milano, 2005. nuova legge istituisce un sistema di controlli più equilibrati, definisce le sanzioni secondo un criterio di progressività e rafforza la propria attività di vigilanza sugli enti locali, al fine di verificare l'efficienza, l'efficacia e la trasparenza dei controlli da loro svolti, sulla base di criteri standard prestabiliti.

Le strutture sono anche tenute alla comunicazione dei flussi turistici secondo le indicazioni regionali e soprattutto all'adempimento della denuncia degli ospiti, in via telematica, come richiesto dalle autorità di pubblica sicurezza. Questa rappresenta una novità per molte tipologie della ricettività che comporterà un lieve carico di lavoro aggiuntivo per i gestori, alleggerito però grazie all'utilizzo esclusivo di procedure di comunicazione telematica ${ }^{23}$.

\section{La revisione delle tipologie di strutture alberghiere}

Come accennato poco sopra, il legislatore lombardo sembrerebbe avere operato secondo una logica di sintesi giuridica spinta soprattutto da un movente di utilità degli istituti. Pertanto ogniqualvolta una tipologia di struttura ricettiva non presenti delle caratteristiche tali da rendere necessaria una disciplina amministrativa a sé stante rispetto a quella generale e valida per tutte le strutture ricettive, che è stata quindi accorpata, non è più presente nell'elenco dell'art. 18; se però, si specifica al comma 5 , al fine di rispondere a particolari esigenze di natura commerciale le strutture caratterizzate da particolari servizi addizionali volessero aggiungere una denominazione che non risulti ingannevole per il turista e non coincida con una di quelle di legge, ma che ne permetta una maggiore riconoscibilità commerciale, lo potrà fare.

È stata così eliminata la bipartizione tra alberghi e residenze turistico-alberghiere che si fondava sulla prevalenza dell'accoglienza in camere o appartamenti, è rimasto il limite delle sette camere e della gestione unitaria per rientrare nella categoria alberghiera, dove è stata aggiunta la tipologia dei condhote ${ }^{24}$. Il condho$t e l$ non è una novità nella realtà giuridica, sebbene sia una formula ancora non molto diffusa nel territorio italiano. Con il decreto "Sblocca Italia", d.l. 12 settem- 
bre 2014 , n. 133 , convertito in 1.11 novembre 2014, n. $164^{25}$, all'art. 31 vengono definiti, nell'ambito delle «Misure per la riqualificazione degli esercizi alberghieri», i condhotel, quali gli esercizi alberghieri aperti al pubblico, a gestione unitaria, composti da una o più unità immobiliari ubicate nello stesso Comune o da parti di esse, che forniscono alloggio, servizi accessori ed eventualmente vitto, in camere destinate alla ricettività $\mathrm{e}$, in forma integrata e complementare, in unità abitative a destinazione residenziale, dotate di servizio autonomo di cucina, la cui superficie non può superare il $40 \%$ della superficie complessiva dei compendi immobiliari interessati. Come prescritto dal comma 3 dello stesso articolo, la Regione Lombardia ha provveduto ad adeguare il proprio ordinamento incorporando senza modifiche la definizione di condhotel all'art. 19, comma 4, salvo specificare che la parte residenziale non potrà in alcun modo beneficiare degli aumenti delle cubature riservate dagli strumenti urbanistici alle superfici destinate a funzioni turistico-ricettive, al fine di evitare comportamenti abusivi o non corretti ${ }^{26}$. Sotto il profilo operativo, attraverso questa nuova forma di ricettività, si permette all'albergatore di vendere (o ai condomini di mettere a disposizione dell'albergatore) camere o appartamenti all'interno della struttura a soggetti privati, che, nel momento in cui non potranno o non vorranno utilizzarli, potranno renderli disponibili per essere oggetto di un contratto di albergo. In una prospettiva privatistica ci si trova di fronte ad un negozio atipico, in cui oltre alla compravendita immobiliare, il soggetto venditore, o un terzo incaricato, si obbliga ad un facere corrispondente alla prestazione di un contratto di albergo, nel limite dei periodi di non occupazione dei locali appartenenti ai diversi proprietari. Pur non essendo questa la sede per un approfondimento, meriterebbe una nota la questione della qualificazione giuridica della fattispecie che non è al momento del tutto chiara. Anche un'eventuale assimilazione dell'istituto alla multiproprietà alberghiera non sarebbe propria, dal momento che il diritto che viene trasferito con questo contratto sembrerebbe essere un pieno diritto di proprietà 27 , che è opinione condivisa non possa corrispondere al diritto trasferito con la multiproprietà.

25. In precedente provvedimento normativi dello stesso anno già si era tentato di inserire i condhotel: d.I. 24 giugno 2014, n. 91

26. A. Cicchetti, I/ vincolo turistico alberghiero: strumento di conservazione o trasformazione del territorio? in Riv giur edil. 2014, p. 150, il quale afferma che andrebbero definiti, anche sotto il profilo penale, i rapporti con la lottizzazione abusiva, per capire se il frazionamento di complessi edilizi autorizzati come residenze turistico

\section{E di quelle non alberghiere}

Anche l'elenco delle strutture non alberghiere è stato sottoposto a revisione. I rifugi alpinistici ed escursionistici sono stati accorpati ai bivacchi fissi, seguendo la logica dell'ubicazione, spariscono gli esercizi di affittacamere, sostituiti dalla "foresterie lombarde", ritenute commercialmente più "accattivanti", e compaiono le "locande".

Le foresterie lombarde acquistano la caratteristica dell'imprenditorialità, incrementano la capacità ricettiva da 12 a 14 posti letto nello stesso numero di stanze (6) (rispetto ai precedenti affittacamere), possono somministrare alimenti e bevande, nel rispetto delle norme igienico sanitarie dei locali e dei prodotti alimentari; possono fregiarsi a proprie spese di un apposito contrassegno identificativo, definito dalla Giunta regionale, quale segno di riconoscimento all'esterno della residenza.

Le nuove locande sono inserite all'articolo 28 nella sezione IV della norma che le disciplina insieme ai bed \& breakfast. Sono strutture ricettive complementari all'esercizio di somministrazione di alimenti e bevande, gestite dallo stesso titolare in forma imprenditoriale in non più di 6 camere, con un massimo di 14 posti letto. $\mathrm{A}$ differenza delle foresterie l'attività deve essere svolta necessariamente in modo unitario nello stesso edificio in cui si svolge l'attività di somministrazione, che quindi è l'attività principale a cui si affianca quella ricettiva. La Giunta definisce un apposito contrassegno per le locande, per permetterne la riconoscibilità commerciale. Tale contrassegna va affisso nei locali, a spese degli operatori. Per quanto concerne le caratteristiche strutturali e igienico sanitarie si distingue tra le esistenti e quelle di nuova costruzione. Le prime possono ancora essere conformi alle norme previste per i locali di civile abitazione, le seconde dovranno, invece, possedere le caratteristiche previste per le strutture ricettive alberghiere.

La disciplina del bed \& breakfast rimane pressoché immutata ma potrà essere esercitata esclusivamente al numero civico di residenza anagrafica del titolare, comprese le pertinenze al fine di evitare fenomeni di abusivismo, in considerazione delle agevolazioni connesse a questo tipo di strutture.

alberghiere e la vendita di singole unità immobiliari destinate a uso abitativo non risulti in contrasto con l'interesse ad un ordinato assetto e sviluppo del territorio.

27. Si veda T. Galletto, Le residenze turistico alberghiere, proprietà frazionata, rapporti condominiali e regolamenti contrattuali, in Foro pad., 2007, p. 130; in tema di multiproprietà si veda G. Tassoni, La multiproprietà, in (a cura di V. Franceschelli - F. Morandi) Diritto del turismo, 2013, p. 332 e ss. 
Sebbene non ne sia stata modificata la definizione, anche le case ed appartamenti per vacanze hanno subito una minima revisione. Permangono la formula imprenditoriale e non imprenditoriale con lo scrimine dell'occasionalità e del limite del numero delle unità abitative (o parti di queste) ora innalzato a tre, in entrambi i casi viene mantenuta la destinazione urbanistica degli alloggi ${ }^{28}$. Le case ed appartamenti per vacanze erano stati inseriti nel codice del turismo all'art. 12 , comma 5 (ora abrogato) sotto la denominazione di "unità abitative ammobiliate ad uso turistico": case o appartamenti, arredati e dotati di servizi igienici e di cucina autonomi, dati in locazione ai turisti, nel corso di una o più stagioni, con contratti aventi validità non inferiore a sette giorni e non superiore a sei mesi consecutivi senza la prestazione di alcun servizio di tipo alberghiero. Rispetto alla norma statale non sono menzionate le caratteristiche funzionali, la cui definizione è rimandata a provvedimenti esterni alla norma, coerentemente con la disciplina degli altri istituti.

\section{La classificazione delle strutture alber- ghiere}

L'annosa questione in merito alla classificazione delle strutture ricettive non sembra ancora avere trovato soluzione a livello nazionale. Il danno di immagine e commerciale, unito al danno alla libera concorrenza, che causa la scarsa affidabilità del rating delle strutture a livello nazionale è un problema che si aggrava sempre di più, quanto più nel resto del mondo ci si dirige verso la standardizzazione e la globalizzazione. Non a caso il successo delle catene alberghiere internazionali si fonda proprio sui valori dell'affidabilità e della soddisfazione delle aspettative del cliente, che prenota e fruisce dei servizi di una struttura nella consapevolezza di ciò che lo aspetta, ed è quindi tutelato dal marchio privato; l'assenza di un rating di tipo statale non può che comportare un abbassamento nei livelli di tutela del turista ${ }^{29}$.

In Italia l'art. 7 della legge $217 / 1983$, prima legge quadro in materia turistica, stabiliva che era compito delle regioni definire i parametri per attribuire una classificazione in stelle alle strutture ricettive sulla base di tre criteri: dimensione della struttura, requisiti strutturali dei servizi offerti e qualificazione degli addetti. Le Regioni

28. Il problema della destinazione degli alloggi, se ad uso abitativo o alberghiero crea uno squilibrio concorrenziale tra strutture alberghiere e strutture non alberghiere, ma svolte a livello imprenditoriale per elevati livelli di capacità ricettiva. definirono ciascuna i propri criteri, partendo dai principi comuni della 217, addivenendo così ad una classificazione molto disomogenea sul territorio nazionale. La questione, sentita da subito come problematica, ha destato l'attenzione del legislatore nazionale che, con la legge di revisione del 13 marzo 2001 n. 135 all'art. $24^{\circ}$ comma ha stabilito che attraverso un decreto del presidente del consiglio dei ministri, da emanare entro tre mesi dalla entrata in vigore della norma, dovevano essere individuati gli standard nazionali omogenei e uniformi per la classificazione. Il d.p.c.m. del 13 settembre del 2002 non ha però provveduto in tal senso, rimandando il lavoro alla Regioni che avrebbero dovuto decidere concordemente. Successivamente nulla di fatto è stato prodotto dalle Regioni, nel frattempo sono intervenuti altri due provvedimenti statali: la legge 344/2007 all'art. 2 comma 193, lett. a, nuovamente rimandava ad un d.p.c.m. per l'armonizzazione degli standard minimi per la classificazione delle strutture ricettive. Il provvedimento del 21 ottobre del 2008 ha identificato standard minimi nazionali dei servizi ricettivi nell'allegato «prospetto di definizione degli standard minimi nazionali dei servizi delle dotazioni per la classificazione degli alberghi», che ha però lasciato alle Regioni l'individuazione di ulteriori criteri collegati alle caratteristiche territoriali di ciascuna. Poche le Regioni che si sono mosse, tanto che il 13 marzo 2013 al punto 19) della delibera della Conferenza permanente Stato-Regioni viene disposta, ai sensi dell'art. 7 comma 2, d.lgs. 28 agosto 1997, n. 281, la costituzione del Gruppo di lavoro in materia di classificazione delle strutture alberghiere per «definire il database sulla base dello schema già predisposto con il repertorio dei criteri individuati dal d.p.c.m. 21 ottobre 2008, dalle normative regionali sui sistemi di classificazione emanate in attuazione del citato decreto, dai sistemi di classificazione adottati dalle maggiori associazioni europee (Hotrec e Hotelstar); 2) Favorire l'aggiornamento periodico dei sistema nazionale di classificazione alberghiero ed extralberghiero in una prospettiva europea; 3) Definire criteri omogenei delle strutture ricettive alberghiere ed extralberghiere e di standard di qualità minimi attraverso un Catalogo volto a definire i servizi e gli standard minimi da offrire ai turisti su tutto il territorio nazionale».

A seguire il d.l. 31 maggio 2014, n. 83 recante «Disposizioni urgenti per riqualificare e migliorare le strutture

29. Per autorevole dottrina la materia della classificazione, nonostante la riforma del titolo $V$ della Costituzione, rimane di competenza statale, in quanto attiene alla disciplina della concorrenza e della tutela del consumatore. Si veda Righi, cit., p. 143; R. Santagata, /l diritto del turismo, Torino, 2014, p. 67 e 68. 
ricettive turistico-alberghiere e favorire l'imprenditorialità nel settore turistico», che al comma 5 statuisce che «per promuovere l'adozione e la diffusione della progettazione universale (...) il Ministero dei beni e della attività culturali del turismo, con proprio decreto da emanare entro tre mesi (...) aggiorni gli standard minimi uniformi in tutto il territorio nazionale, dei servizi e delle dotazioni per la classificazione delle strutture ricettive e delle imprese turistiche, (...) tenendo conto delle specifiche esigenze connesse alla capacità ricettiva e di fruizione dei contesti territoriali e dei sistemi di classificazione alberghiera adottati a livello europeo ed internazionale». Il decreto, purtroppo, non è mai stato emanato, mentre nuovamente a livello nazionale con il decreto Sblocca Italia, si è ribadita la necessità, rimandando ad un nuovo provvedimento (un d.p.c.m.) con la stessa finalità. Nel vuoto normativo a livello centrale, sensibile alla necessità di un adeguamento comunque nazionale se non addirittura europeo e, soprattutto, di un superamento dell'attuale sistema, la Regione Lombardia ha stabilito che la Giunta predisponga, anche in coordinamento con le altre regioni e lo Stato, un sistema di classificazione omogenea sul territorio che tenga anche conto degli orientamenti internazionali. La vera novità riguarda però la possibilità di attribuire alle strutture una valutazione integrativa che individui i servizi offerti in aggiunta a quelli standard, che tenga conto della data di costruzione o di ultima ristrutturazione dell'edificio. La valutazione integrativa va esposta all'interno dell'albergo, ed eventualmente all'esterno cercando di darne massima visibilità, mentre la classificazione standard deve essere visibile sia all'esterno sia all'esterno della stessa. L'obiettivo del legislatore è quindi quello di sopperire ad un endemico difetto in materia di classificazione, cercando di raggiungere il più possibile,

30. Una prima disciplina organica a livello statale delle professioni turistiche, si è avuta con la legge quadro $217 / 83$, la quale all'articolo 11 , per la prima volta più che una definizione generale e astrattamente applicabile, dava l'elenco delle diverse professioni, specificando poi che le singole Regioni, oltre a dovere accertare i requisiti per potere svolgere le diverse attività e definire la disciplina di dettaglio, avrebbero potuto incrementare le figure professionali in funzione delle particolarità del proprio territorio e degli sviluppi del mercato, sempre tenendo conto dell'interesse del fruitore finale. L'elenco della 217/83 per lunghissimo tempo ha rappresentato il punto di rifermento per la disciplina delle professioni e per l'individuazione delle diverse tipologie.

31. Si veda Corte Cost. n. 222 del 2008; n. 271 del 2009; n. 312 del 2010.

32. La Corte Costituzionale con la sentenza n. 222 del 2008 ha inquadrato le professioni turistiche nella materia professioni e non turismo. Secondo la Corte, infatti, la tutela del turista giustifica l'esclusiva competenza dello stato sull'individuazione di profili professionali e necessari per il relativo esercizio: Corte Costituzionale 29 ottobre 2009 n. 271; Corte Costituzionale 15 aprile 2010 n. 132. Alle Regione compete la sola formazione dei professionisti.

In dottrina, tra gli altri: L. Righi, Professioni turistiche tra Stato tra Stato, Regioni, e Corte Costituzionale: si stava meglio quando si stava peggio ? in Nuovo dir. tur. 2/20111, p. 67; M.E. La Torre, II ruolo della guida turistica per la conoscenza del territorio. Profili civilistici, in Nuovo dir. tur., 3/2011, p. 7. anche attraverso ulteriori criteri, l'obiettivo della effettiva rappresentatività del livello attribuito. La classificazione ottenuta dalle strutture non rappresenta, infatti, una conquista definitiva. Se prima il numero di stelle attribuite aveva una durata di 5 anni, oggi la classificazione va aggiornata ogni qualvolta vi siano dei cambiamenti. La Provincia a le città Metropolitana di Milano, infatti, svolgono delle verifiche a campione per controllare la fondatezza della classificazione ottenuta e pubblicizzata, nonché un sistema di sanzioni (artt. 39 e 40) al fine di evitare abusivismi.

\section{Le professioni turistiche}

Sotto il profilo delle figure professionali la norma recepisce la legge statale n. 97 del 2013 che all'articolo 3 ha attribuito valenza nazionale alla professione di guida turistica. Per quanto attiene alle professioni turistiche che sono quelle «(...) aventi ad oggetto la prestazione di servizi di promozione dell'attività turistica, nonché servizi di ospitalità, assistenza, accompagnamento e guida, diretti a consentire ai turisti la migliore fruizione del viaggio e della vacanza, anche sotto il profilo della conoscenza dei luoghi visitati» (art. 6 cod. tur.) non semplice è stato il percorso di attribuzione delle competenze Stato-Regione: la disciplina delle professioni ${ }^{30}$ è oggi materia concorrente, non è pertanto incidentale che l'articolo 6 del codice del turismo sia sopravvissuto alla censura costituzionale ${ }^{31}$.

La questione si pone sul piano autorizzatorio: professioni protette o non protette e soprattutto sulla validità territoriale del titolo ${ }^{32}$. Oggi, dapprima grazie all'art. $3,5^{\circ}$ comma d.l. 138/2011, l. 14 settembre 2011, n. $148^{33}$, l'accesso alle professioni non protette è libero. In base a

33. Art. 3 Comma 1: "Comuni, Province, Regioni e Stato, entro un anno dalla data di entrata in vigore della legge di conversione del presente decreto, adeguano i rispettivi ordinamenti al principio secondo cui l'iniziativa e l'attività economica sono libere ed è permesso tutto ciò che non è espressamente vietato dalla legge nei soli casi di: a) vincoli derivanti dall'ordinamento comunitario, c) contrasto con i principi fondamentali della Costituzione; c) danno alla sicurezza, alla libertà, alla dignità umana e contrasto con l'utilità sociale; d) disposizioni indispensabili per la protezione della salute umana, la conservazione delle specie animali e vegetali, dell'ambiente, del paesaggio e del patrimonio culturale (...)»; comma 5: «Gli ordinamenti professionali devono garantire che l'esercizio dell'attività risponda senza eccezioni ai principi di libera concorrenza, alla presenza diffusa dei professionisti su tutto il territorio nazionale, alla differenziazione e pluralità di offerta che garantisca l'effettiva possibilità di scelta degli utenti nell'ambito della più ampia informazione relativamente ai servizi offerti, sono vietate le restrizioni alla libera prestazione dei servizi all'interno dell'Unione». "La possibilità delle region di creare e disciplinare professioni turistiche, ulteriori o anche "specificative" di quelle previste dalla legislazione statale sembra tuttavia oggi essere messa in dubbio dall'assetto costituzionale del riparto di competenze in materia di professioni turistiche frutto della riforma del Titolo $V$ della Carta Costituzionale, così come è stato interpretato recentemente dalla Corte Costituzionale.» Così L. Righi, cit., p. 187. Diverse legislazioni regionali hanno però disciplinato figure professionali 
quanto disposto dall'art. 3, legge 6 agosto 2013 , n. 97 , la professione di guida turistica è libera, e, in ottemperanza del principio comunitario, la Regione Lombardia nell'attuale norma ha disciplinato l'accesso alle attività di guida e accompagnatore richiamando esplicitamente i principi nazionali e comunitari, rimandando al provvedimento di Giunta la disciplina di dettaglio in merito all'abilitazione, coerentemente con gli altri ambiti di intervento normativo. Nello stesso solco si inserisce l'articolo 51 sui siti di particolare interesse storico, per cui è prevista una disciplina $a d$ hoc delle professioni a maggior tutela di un interesse generale. La previsione di un particolare titolo abilitativo per quanto riguarda la professione di guida nei siti di "specializzazione" che tenga conto delle disposizioni nazionali e comunitarie è il risultato di un lungo periodo di giochi di forza tra Regioni ed Unione Europea soprattutto sotto il profilo dell'individuazione dei siti di particolare interesse che meritassero una speciale tutela, quindi l'abilitazione di guide specializzate ${ }^{34}$.

Come è noto diversi sono stati i richiami alle Regioni per la disciplina delle professioni. La Corte di giustizia della comunità europea è intervenuta a più riprese per dichiarare incompatibili con le norma comunitarie le restrizioni in materia di circolazione delle guide turistiche $^{35}$; anche la Corte di Cassazione, 18 maggio 2006, n. 11751, ha ribadito che le guide stabilite in un Paese Membro dell'Unione Europea diverso dall'Italia e che accompagnano un gruppo di turisti provenienti dallo stesso Paese membro, nel corso di un viaggio organizzato con durata limitata nel tempo ed a circuito chiuso, possono esercitare la suddetta attività anche in assenza della prescritta autorizzazione; ed ha disapplicato, perché in contrasto con l'articolo 56 TFUE, la L.R. Veneto del 2003 n. 33 nella parte in cui riservava inderogabil-

nuove o "specificative": Regione Calabria: 5 aprile 2008 n. 8 artt. 31-38, guida naturalistico ambientale, animatore del patrimonio: è guida naturalistico ambientale chi esercita professionalmente l'attività di conduzione di persone nelle visite a parchi, riserve naturali, zone di pregio o tutela ambientale o siti di interesse ambientale così come individuate dalla legislazione vigente, fornendo notizie e informazioni di interesse naturalistico, paesaggistico ed ambientale; Friuli Venezia Giulia: I.r. n. 2 del 16 gennaio del 2002 (numerose revisioni): guida naturalistica o ambientale; Liguria: I.r. 23 dicembre 1999 n. $44 \ldots$ guida ambientale ed escursionistica; Marche: I.3 9/2006 guida naturalistica; Sardegna, I.r. 20 del 18 dicembre 2006, disciplina per le modalità attuative degli esami per l'abilitazione all'esercizio delle professioni di (...) guida ambientale escursionistica «Ė guida ambientaleescursionistica chi per professione accompagna persone singole o gruppi di persone in ambienti terrestri o acquatici, compresi parchi ed aree protette, illustrandone le peculiarità paesaggistiche, naturalistiche faunistiche, botaniche e geologiche».

34. Gli organi comunitari hanno rimesso ai singoli Stati Membri la facoltà di individuare "peculiari contesti" riservati alle "guide specializzate" e l'Italia con il d.P.R 13 dicembre 1995 (considerato soddisfacente anche dalla Commissione europea) demandava alle Regioni, d'intesa con le Sovrintendenze, l'individuazione dei siti, per i quali, quindi l'attività di guida turistica svolta da soggetti provenienti da paesi esteri era sottoposta ad un provvedimento di riconoscimento di validità del titolo in Italia, secondo quanto stabilito dal d. Igs. n. 319 del 1994. I siti individuati furo- mente alle guide specializzate munite di apposita licenza rilasciata dal Comune, l'illustrazione ai visitatori dell'intera città di Venezia. Una volta permesso però il libero ingresso e la libera "professionalità" ai cittadini europei, la sperequazione normativa dall'ambito comunitario si spostava a quello interregionale: paradossalmente i cittadini comunitari, ma di nazionalità differente, avrebbero potuto operare su tutto il territorio italiano, mentre i cittadini italiani avrebbero dovuto rimanere operativi solo sul proprio territorio regionale. Immediato l'intervento della Corte costituzionale, che, con la sentenza n. 271 del 2009, ha stabilito l'illegittimità delle restrizioni territoriali interne all'attività, statuendo la libera circolazione dei professionisti all'interno di tutto il territorio nazionale. Principio di libera prestazione dei servizi che, come si è detto poc'anzi, è stato poi normato all'articolo 3 legge $97 / 2013$, che sancisce al contempo l'unico temperamento al principio assegnando al Ministero per i beni e le attività culturali ed il turismo, sentita la Conferenza Unificata Stato-Regioni, l'indicazione in un decreto i siti nei quali lo svolgimento di guida turistica è riservato ${ }^{36}$. Sul fondamento del suddetto principio è consentito l'inserimento dell'articolo 51 all'interno della legge lombarda, che appunto ha operato un esplicito adeguamento ai principi comunitari e nazionali della libera circolazione delle professioni, senza sottovalutare l'imprescindibile salvaguardia dell'interesse del turista per un'informazione corretta, attuale e completa.

\section{Le agenzie di viaggio e turismo}

L'articolo 57 definisce le agenzie di viaggio e turismo le imprese che esercitano in via principale, attività di pro-

no 2.500 coincidenti con quelli considerati dall'UNESCO patrimonio generale delI'Umanità. Oltre all'elevato numero, i siti spesso coincidevano con intere città (Venezia, Verona, Vicenza...) e non con singoli luoghi di interesse. Per questo motivo nel 2003 I'Unione europea ha aperto una procedura di infrazione, che si è chiusa quattro anni più tardi con la legge 2 aprile 2007, n. 40, che ha dichiarato che «i soggetti abilitati allo svolgimento di guida turistica nell'ambito del Paese comunitario di appartenenza, operano in regime di libera prestazione dei servizi, senza necessità di alcuna autorizzazione né abilitazione, sia essa generale o specifica». La riapertura alle guide di siti specializzati si ha con la legge 97/2013 che ha temperato il principio della libera circolazione delle professioni con la previsione dell'identificazione da parte del Ministero dei beni culturali, di un elenco de siti si specializzazione. Nel 2015 si sono avuti i decreti "Franceschini", così chiamati dal nome del Ministro: 29 gennaio 2015 e 17 aprile 2015.

35. 26 febbraio 1991 in Causa C 180/89. 22 marzo 1994 in causa C-375/92: 5 giugno 1997 in causa C-398/95. II principio comunitario vieta tutte quelle restrizioni che risultino essere sproporzionate rispetto all'obiettivo di pubblico interesse, obiettivo (di tutela del patrimonio storico-artistico) dietro il quale I'Italia si è celata proprio per potere portare avanti il regime di restrizioni alla professione turistica.

36. I decreti sono arrivati nel 2015: decreto "Franceschini" 29 gennaio 2015 e 17 aprile 2015. 
duzione, organizzazione, prenotazione e vendita di biglietti di viaggi, soggiorni, ovvero intermediazione nei predetti servizi o entrambe le attività. Con la nuova definizione, in linea con le norme di disciplina del contratto di viaggio, sparisce la bipartizione tra agenzia travel agent e agenzia tour operator, ma vengono ampliate le attività che a queste possono competere. All'art. 57 comma 4 si rimanda ad una deliberazione di Giunta per stabilire gli eventuali compiti aggiuntivi che potranno svolgere le $\mathrm{AdV}$, ma al contempo si specifica già in questa sede che, rispetto al passato, ora potranno effettuare attività di incoming e di informazione ed accoglienza turistica. Nella norma è stato eliminato ogni riferimento al contenuto del programma di viaggio (art. 88 l.r. 15/2007), poiché trattandosi di una disposizione già contenuta nelle poche norme di natura civilistica sopravvissute del codice del turismo e cioè quelle relative ai contratti del turismo organizzato, art. 32 e ss., una ripresa in questa sede sarebbe stata pleonastica. Al comma 2 dell'articolo 57 viene riconosciuta l'operatività on-line delle agenzie, del pari però è stabilito al quinto comma dell'articolo seguente, che, nel caso in cui la sede dell'agenzia on-line si trovi in uno Stato diverso da quello italiano, a tutela del turista, il titolare dell'attività ha l'obbligo di indicare il responsabile della stessa per il territorio della Lombardia. Si tratta di un'interessante novità che tenta, per quanto possibile, di offrire una garanzia maggiore al turista. Non può non dirsi che si tratta di un provvedimento assi meritevole che però, purtroppo, come spesso accade nelle contrattazioni via $w e b$, ha una portata di tutela che per ragioni di natura "tecnologica" non è in grado di coprire e garantire la protezione del turista ogniqualvolta in cui questi acquisti on-line, trovandosi su suolo lombardo, ma da agenzie on-line con sede all'estero. La tutela del turista rientra tra gli obiettivi della norma che infatti prevede all'articolo 61 che le AdV stipulino, prima della presentazione della SCIA ${ }^{37}$, congrua polizza assicurativa a garanzia dell'esatto adempimento degli obblighi assunti verso i clienti con il contratto ed i programmi di viaggio e in relazione al costo complessivo dei servizi offerti, nell'osservanza delle disposizioni previste in materia della normativa nazionale in vigore. Gli obblighi assicurativi non riguardano le sole $\mathrm{AdV}$, ma anche gli Organizzatori di viaggi diversi da adv art. 66 e per le Associazioni, gruppi sociali e comunità art. 67. Gli articoli 66 e 67 disciplinano tutti i casi in cui i soggetti che svolgono funzioni di agenzia non siano imprese turistiche, ma altre tipologie di enti (ipotesi già presenti nel precedente articolato, artt. 95 e 96 con una disciplina simile). Nel caso ci si trovi di fronte ad associazioni, ipotesi disciplinata anche e nel codice del turismo, si statuisce che gli enti senza scopo di lucro (che non presentano una SCIA) che operano per finalità ricreative, culturali, religiose e sociali e che siano riconosciute formalmente a livello nazionale possono svolgere l'attività di organizzazione, realizzazione di viaggi e soggiorni in modo continuativo, secondo gli scopi statutari, esclusivamente per i propri associati e, soprattutto, nel rispetto di quanto stabilito agli articoli 32 e ss. del Codice del Turismo, qui espressamente ed eccezionalmente richiamato. All'art. 67 i soggetti che organizzano viaggi, soggiorni e servizi turistici sono sempre enti, associazioni, gruppi sociali, comunità ed enti concordatari con finalità politiche, sociali, sindacali, religiose, culturali o sportive senza scopo di lucro, ma a differenza di quelli disciplinati all'articolo sopra, svolgono l'attività turistica in modo occasionale, per questi quindi non è previsto l'assoggettamento alle disposizioni della norma, ma come già accennato sono tenuti all'obbligo assicurativo per i rischi derivanti ai partecipanti, stipulano una polizza di responsabilità civile, in mancanza della quale oltre alla sanzione pecuniaria, è prevista la cessazione di ogni ulteriore attività.

\section{Il rinnovato ruolo delle Camere di com- mercio}

Le Camere di Commercio (CCIAA) hanno sempre avuto un ruolo di rilievo per quanto attiene al settore del turismo. Nella precedente Legge lombarda 15/2007, infatti, oltre ad essere loro interamente dedicato l'art. 11, nel quale venivano esplicitamente indicate le funzioni delle camere nel turismo (promozione e sostegno alla costituzione di nuove imprese; formazione; tenuta albi; azioni per la tutela dei diritti del turista...), venivano menzionate anche all'articolo 9, comma 2 quali enti preposti all'istituzione degli uffici di informazione ed accoglienza turistica, all'articolo 28 comma 4, per il rilascio di licenze di esercizio per le aziende alberghiere in presenza di denominazione identica a quella di altre aziende operanti nel territorio dello stesso comune, all'art. 91 sempre per il rilascio dei certificati attestanti la natura 
e la durata delle attività svolte in maniera indipendente dai direttori tecnici.

$\mathrm{Nel}$ generale contesto di riforma delle Camere con il nuovo testo normativo il loro ruolo nell'ambito del comparto turistico sembra consolidarsi rinnovandosi.

Lart. 9 (Partenariato delle CCIAA) ad esempio non ripropone l'elenco delle funzioni di loro competenza, ma le investe del ruolo chiave di promotori dello sviluppo di progetti ed iniziative per l'attrattività turistica e integrata del territorio di riferimento, con l'obiettivo del consolidamento e della crescita competitiva e qualitativa delle imprese. All'articolo 5 viene confermato il compito istituzionale relativo alle commissioni arbitrali e conciliative e all'articolo 6 il ruolo di enti di cui possono avvalersi le province e la Città Metropolitana di Milano, d'intesa con la Regione e nel rispetto delle norme previste dalla legge. Partecipano, senza oneri per la Regione all'Osservatorio regionale del turismo (art. 14) ed infine mantengono il loro ruolo legato alla formazione professionale (art. 77).

\section{Conclusioni}

La presente legge costituisce un interessante passo avanti per lo sviluppo del turismo in Lombardia. La Regione Lombarda per anni non ha rappresentato uno dei principali poli dell'attrattività turistica in Italia, sopraffatta da luoghi di maggior interesse storico, culturale, balneare... rappresentava, invece, trainata dal capo- luogo, un importante territorio di sviluppo industriale. Grazie a politiche di espansione dell'attrattività turistica (e nell'anno attuale anche all'esposizione universale) è riuscita negli ultimi anni a sviluppare il proprio potenziale, non soltanto in ambiti, già turisticamente più rilevanti, quali i comprensori montani, i laghi, le città storiche, ma anche in contesti per anni considerati di scarso interesse turistico, quali Milano. Il valore della recente norma è di avere focalizzato l'obiettivo sulla valorizzazione del capitale umano e del patrimonio esistente cercando di riqualificarlo il più possibile, e al contempo di semplificare quanto più le modalità di accesso al settore per gli operatori, eliminando inutili adempimenti di natura burocratica e inutili provvedimenti normativi, tesi più a confondere che a chiarire, senza dimenticare l'obiettivo della tutela del turista (ivi compresi i turisti disabili). Lavere esplicitamente allargato il concetto di impresa turistica a qualsiasi tipologia di impresa della filiera, avere fortemente potenziato le collaborazioni pubblico-privato, cercando di imporre le logiche del privato nel pubblico e non il viceversa (l'operatività viene misurata in termini di efficacia ed efficienza e solo in base ai risultati premiate le iniziative di valore), avere legato il marchio lombardo a quello italiano nella promozione all'estero, avere spinto per la digitalizzazione dei processi e della raccolta delle informazioni, avere previsto una pianificazione della politica turistica che misuri i risultati conseguiti e, soprattutto, che ne tenga conto per le politiche future, rappresenta una valida partenza che andrà via via consolidata. 